Check for updates

Cite this: RSC Adv., 2018, 8, 8937

Received 30th October 2017 Accepted 5th February 2018

DOI: 10.1039/c7ra11937b

rsc.li/rsc-advances

\title{
The hydrothermal synthesis of ultra-high aspect ratio Ag nanoflakes and their performance as conductive fillers in heaters and pastes $\uparrow$
}

\author{
Hua Wang, ${ }^{\text {ab }}$ Wenjuan Yang, ${ }^{\text {ab }}$ Kaibin Li ${ }^{\mathrm{ab}}$ and Guanghai Li (D) *a \\ Ag nanoflakes with a size ranging from 5 to $60 \mu \mathrm{m}$, a thickness of several tens of nanometers and an aspect \\ ratio of up to 800 have been synthesized via a hydrothermal method. PVP was used as both a surfactant, \\ inducing anisotropic growth of the Ag nanoflakes, and as a reductant, reducing $\mathrm{Ag}^{+}$to $\mathrm{Ag}$. $\mathrm{An} \mathrm{Ag}-$ \\ oxalate complex was used as a precursor, allowing effective control of the kinetic growth of the $\mathrm{Ag}$ \\ nanoflakes. Influences on the size and morphology of the Ag nanoflakes, such as $\mathrm{H}^{+}$concentration and \\ reaction time, were discussed and analyzed. Our method can be easily scaled up for mass production. A \\ large interfacial contact area between the Ag nanoflakes with more electrical channels makes the $\mathrm{Ag}$ \\ nanoflakes excellent conductive fillers.
}

\section{Introduction}

Due to their unique physical and chemical properties, silver nanomaterials have attracted extensive attention in fields such as optoelectronics, ${ }^{1-4}$ optics, ${ }^{5,6}$ SERS,${ }^{7-11}$ conductive pastes, ${ }^{12-16}$ and heaters. ${ }^{17,18}$ It was found that morphology, size, and crystallinity strongly affect the performance of $\mathrm{Ag}$ nanomaterials, and great efforts have been made towards obtaining Ag nanostructures with specific morphologies such as dendrites, ${ }^{19}$ nanowires, ${ }^{20}$ nanorods, ${ }^{21}$ nanocubes, ${ }^{22}$ and nanoflakes. ${ }^{23} \mathrm{Ag}$ nanoflakes with a high aspect ratio have been widely studied because of their potential applications as SERS-active substrates and as high conductivity fillers in Ag conductive pastes.

$\mathrm{Ag}$ nanoflakes can be prepared via either physical or chemical methods. Physical methods, like the ball-milling method, ${ }^{24}$ can be used to prepare $\mathrm{Ag}$ nanoflakes, but the nanoflakes have poor crystallinity and a rough surface. Chemical methods, such as electrochemical methods, ${ }^{25}$ ultrasonic radiation, ${ }^{26}$ solvothermal methods, ${ }^{27}$ direct chemical reduction, ${ }^{28}$ and photoinduced chemical methods, ${ }^{29}$ have been widely used to synthesize $\mathrm{Ag}$ nanoflakes. A solvothermal method has been used to fabricate $\mathrm{Ag}$ nanoplates by reducing $\mathrm{AgNO}_{3}$ with $\mathrm{N}, \mathrm{N}$ dimethylformamide in the presence of PVP at a high temperature of $160{ }^{\circ} \mathrm{C},{ }^{27}$ but the $\mathrm{Ag}$ nanoplates usually have a low aspect ratio. A chemical reduction method has been developed to synthesize $\mathrm{Ag}$ nanosheets using hydrogen peroxide as

${ }^{a}$ Key Laboratory of Materials Physics, Anhui Key Laboratory of Nanomaterials and Nanostructures, Institute of Solid State Physics, Chinese Academy of Sciences, Hefei 230031, PR China. E-mail: ghli@issp.ac.cn

${ }^{b}$ University of Science and Technology of China, Hefei, 230026, PR China

$\dagger$ Electronic supplementary information (ESI) available. See DOI: $10.1039 / \mathrm{c} 7 \mathrm{ra11937b}$ a reducing agent at room temperature,$^{30}$ and the $\mathrm{Ag}$ nanosheets have a lateral edge length of up to $15 \mu \mathrm{m}$ and a thickness of $28 \mathrm{~nm}$. Plate-like $\mathrm{Ag}$ with an edge length of tens of micrometers has been prepared using chloroplatinic acid as a catalytic agent, in a solution of ethylene glycol and ammonia, in the presence of Pt seeds. ${ }^{31}$ Micrometer sized ultra-thin Ag nanosheets have also been synthesized using $\mathrm{Cu}^{+}$as a reducing agent in an organic solvent containing oleylamine..$^{32}$

The growth of $\mathrm{Ag}$ nanoflakes is a kinetically controlled process, and maintaining a low $\mathrm{Ag}$ concentration in the reaction system is essential to suppress the self-nucleation of Ag. ${ }^{23,33} \mathrm{The}$ crystal morphology and size of the Ag nanomaterials can be well controlled by adjusting the crystal nucleation and growth in either a thermodynamically or kinetically controlled process. The growth of Ag nanoflakes is mainly a kinetically controlled process, and the morphology of the Ag crystal nucleus is determined by its surface energy. The surface energy has the relationship: $\gamma\{111\}<\gamma\{100\}<\gamma\{110\}$ for FCC Ag, and thus the $\{111\}$ facet is generally the crystal facet found in nucleation and growth under kinetic conditions, in which the most stable structure is a stacking fault with $\{111\}$ facets. It was found that the stacking fault is essential in inducing the growth of the $\mathrm{Ag}$ nanoflakes, ${ }^{34,35}$ while a slow reduction speed of $\mathrm{Ag}^{+}$to $\mathrm{Ag}$ is beneficial for obtaining the stacking faults. The stacking faults in the $\{111\}$ crystal surface can form reentrant groove structures on the six sides of the Ag crystal, which will provide nucleation sites in the initial growth stage, and allow the region to grow rapidly to a certain thickness which energetically facilitates subsequent lateral crystal growth. PVP can be used as both a surfactant and a reductant because of its special molecular structure with a lactam group. ${ }^{36-38} \mathrm{Ag}$ twin seeds with stacking faults formed at an initial stage can anisotropically grow into Ag nanoflakes in an aqueous colloidal system of PVP, ${ }^{39}$ and under 
well-controlled conditions, regular hexagonal Ag nanoflakes have been obtained. ${ }^{40}$

In this work we report a hydrothermal synthesis of $\mathrm{Ag}$ nanoflakes using an Ag oxalate ligand as a precursor and PVP as both the capping and reducing agent. Ag nanoflakes with different morphologies such as triangular, truncated triangular, hexagonal and dodecagonal, and a high aspect ratio were obtained. Our method can effectively harness the kinetic growth process of the Ag nanoflakes and can be easily scaled up for mass production. The applications of the $\mathrm{Ag}$ nanoflakes as highly conductive fillers in film heaters and in commercial Ag paste were demonstrated.

\section{Results and discussion}

The growth process of $\mathrm{Ag}$ crystals is very complicated under kinetic conditions because of the influence of factors such as temperature disturbances and the surfactant, resulting in the formation of Ag nanoflakes with different shapes. Fig. 1 shows the FESEM images of the Ag nanoflakes in the same reaction system. One can clearly see the Ag nanoflakes with different shapes, such as triangular, hexagonal and dodecagonal. It is worth noting that, in spite of their different shapes and sizes, almost all the Ag nanoflakes have a good crystal symmetry. It was found that the dominating shape of the Ag nanoflakes depends strongly on the growth conditions and the components of the reaction system. Fig. S1a $\uparrow$ shows a typical XRD pattern of the Ag nanoflakes, in which there exists a solo strong sharp peak situated at about $38.1^{\circ}$, demonstrating a high crystallinity and a preferred orientation of growth along the [111] direction of the $\mathrm{Ag}$ nanoflakes. The corresponding FESEM image shows that the $\mathrm{Ag}$ nanoflakes have a relatively uniform size with an average edge length of about $35.2 \mu \mathrm{m}$ (see the inset of Fig. S1a†). Fig. S1b $\uparrow$ shows a typical TEM image of a triangular Ag nanoflake and the corresponding SAED pattern. The presence of $1 /$ $3(-422)$ and $2 / 3(-422)$ diffraction spots, which are normally forbidden in a FCC structure, provides evidence of the presence of stacking faults in the Ag nanoflake. It was found the size of Ag nanoflakes depends strongly on the experiment parameters like

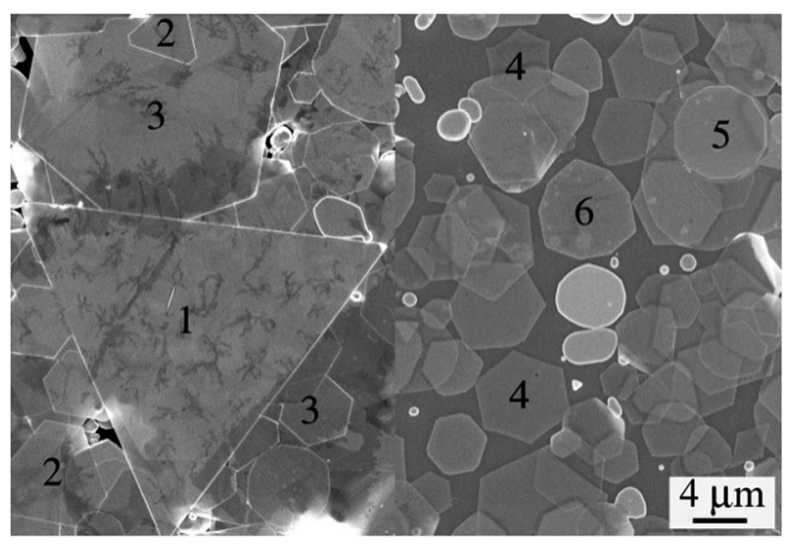

Fig. 1 FESEM images of Ag nanoflakes with different shapes: (1) triangular, (2) truncated triangular, (3) hexagonal, (4) distorted hexagonal, (5) dodecagonal and (6) nonagonal in the same reaction system. the polymerization degree and concentration of $\mathrm{PVP}, \mathrm{H}^{+}$ concentration, reaction temperature and time. To realize the controllable synthesis of $\mathrm{Ag}$ nanoflakes, the optimizing of these parameters is essential.

Fig. 2 shows the AFM profiles of triangular Ag nanoflakes. One can see nanoflakes with edge lengths of $11,16.2$ and 18.7 $\mu \mathrm{m}$, thicknesses of 31,36 and $53 \mathrm{~nm}$ and aspect ratios of 355 , 450 and 353 , respectively. Clearly, the thickness increases with an increasing edge length of the $\mathrm{Ag}$ nanoflakes. The growth of the $\mathrm{Ag}$ nanoflakes is a time-dependent process, and although the deposition rate of $\mathrm{Ag}$ onto the side facets of the flakes is much faster than that onto the $\{111\}$ facets because of the selective adsorption of the surfactant, PVP-K60, the thickness of the Ag nanoflakes will increase with increasing lateral growth. The nanoflakes depend strongly on the experimental parameters, such as the polymerization degree and concentration of PVP, the $\mathrm{H}^{+}$concentration, the reaction temperature and time. To realize the controllable synthesis of Ag nanoflakes, optimization of these parameters is essential.

\section{Polymerization degree and concentration of PVP}

As a surfactant, PVP can preferentially adhere onto the $\{111\}$ crystal planes because the carbonyl $-\mathrm{C}=\mathrm{O}$ with a lone pair of electrons can easily coordinate with $\mathrm{Ag}$ and play a key role in determining the size and morphology of the Ag nanoflakes. PVP is also a weak reductant because of the relatively strong electronegativity of $\mathrm{O}$ and $\mathrm{N}$ atoms of the lactam group, ${ }^{39}$ in which the electron cloud will tend towards the $\mathrm{O}$ atom in carbonyl group and the carbonyl-linked $\mathrm{N}$ atom. This weak reducing power can maintain the free $\mathrm{Ag}$ atoms in the reaction system at a consistently low concentration, facilitating the growth of the $\mathrm{Ag}$ atoms in a hexagonal closely packing way, which is the
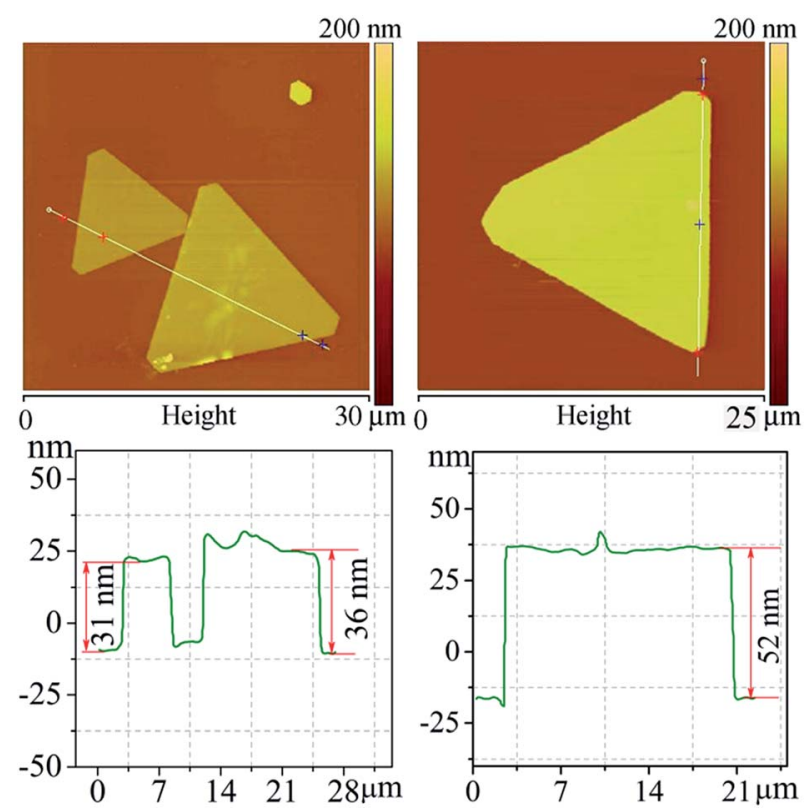

Fig. 2 The AFM images and the corresponding height profiles of triangular Ag nanoflakes. 

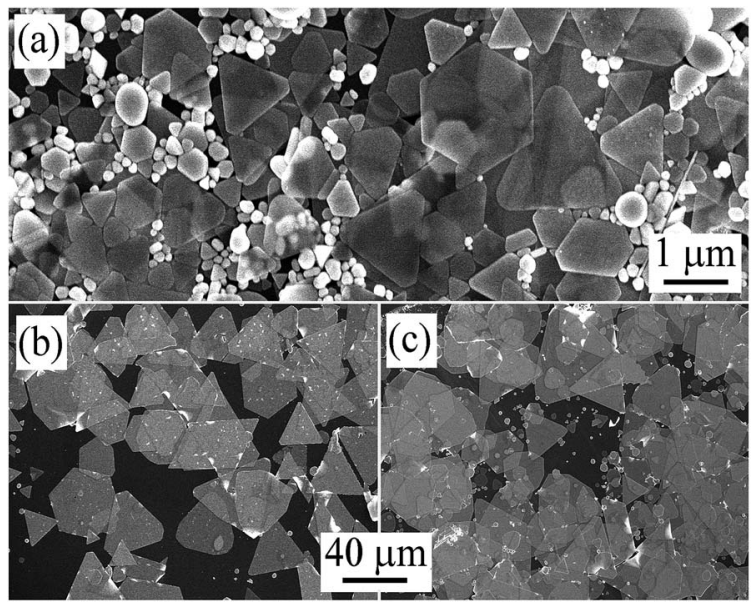

Fig. 3 FESEM images of Ag nanoflakes with a PVP polymerization degree of (a) 30, (b) 60 and (c) 90

reason for the formation of stacking faults in the Ag seed crystals. Fig. 3 shows the FESEM images of the Ag nanoflakes grown with different PVP polymerization degrees (with the same mass content in the reaction systems). One can see that the size of the $\mathrm{Ag}$ nanoflakes increases with an increase in the PVP polymerization degree, and that the average size of the $\mathrm{Ag}$ nanoflakes is about $1.21,35.2$ and $38.6 \mu \mathrm{m}$ with PVP-K $=30,60$ and 90, respectively. As the chain length of PVP-K30 is shorter than PVP$\mathrm{K} 60$ and $-\mathrm{K} 90$, the resultant $\mathrm{Ag}$ nanoflakes have a smaller lateral dimension, see Fig. 3a. Also, there are many spherical Ag particles with PVP-K $=30$ and some irregular particles with PVP$\mathrm{K}=90$, see Fig. $3 \mathrm{a}$ and $\mathrm{c}$. The Ag nanoflakes grown with PVP-K $=$ 60 mainly have triangular and hexagonal shapes, see Fig. 3b, while those grown with PVP-K $=90$ have triangular, hexagonal and enneagonal shapes, see Fig. 3c. The formation of the spherical $\mathrm{Ag}$ particles is due to a complete coverage of the $\mathrm{Ag}$ seed planes with excess PVP-K $=30$ molecules, while that of the irregular particles is caused by the self-nucleation of $\mathrm{Ag}$ with less PVP-K $=90$ molecules; the causes of these phenomena will be discussed below. The above results indicate that the PVP polymerization degree affects both the size and the shape of the Ag nanoflakes.

The influence of the amount of PVP-K $=60$ on the size of the $\mathrm{Ag}$ nanoflakes was further studied. Fig. S2 $\uparrow$ shows FESEM images of Ag nanoflakes with different PVP-K $=60$ concentrations. One can see that the size of the Ag nanoflakes increases with increasing PVP-K $=60$ concentration. There will be an excessive amount of $\mathrm{Ag}$ in the reaction system if the PVP concentration is too low (with the addition of $0.09 \mathrm{M}$ ), leading to the formation of irregular $\mathrm{Ag}$ nanoparticles due to the selfnucleation of free $\mathrm{Ag}$, as shown in Fig. S2a. $\dagger$ However, if the PVP concentration is too high (with the addition of $0.63 \mathrm{M}$ ), all the $\mathrm{Ag}$ seed surfaces will be covered by the PVP, leading to the formation of spherical $\mathrm{Ag}$ nanoparticles due to isotropic growth, see Fig. S2g. $\dagger$ It was found that different sized Ag nanoflakes and small Ag nanoparticles can be obtained with a PVP-K60 concentration in the range of $0.18-0.36 \mathrm{M}$, see Fig. S2b-f. $\dagger$

\section{$\mathrm{H}^{+}$concentration $\left(C_{\mathrm{H}^{+}}\right)$}

An Ag seed nucleus with a stacking fault structure is essential in the formation of Ag nanoflakes,$^{35}$ and such seeds can be formed only under certain conditions, and if the reaction system contains an oxidation etchant, this will be unfavourable for the formation of Ag seeds with stacking faults. In this study, an Agoxalate complex was used as a precursor to effectively control the $\mathrm{Ag}$ ion concentration, which is beneficial for the kinetically controlled growth of the $\mathrm{Ag}$ nanoflakes. As a strong oxidant, $\mathrm{HNO}_{3}$ can be used to etch the high-energy planes of an Ag crystal nucleus with stacking faults, whose oxidability can be effectively adjusted by adding $\mathrm{Na}_{2} \mathrm{C}_{2} \mathrm{O}_{4}$. Since $\mathrm{NO}_{3}{ }^{-}$and $\mathrm{H}^{+}$exist simultaneously in the reaction system, we can take $\mathrm{H}^{+}$ concentration as an indicator to describe the oxidation properties. Fig. 4 shows the FESEM images of the products with different $\mathrm{H}^{+}$concentrations (different amounts of added $\mathrm{Na}_{2} \mathrm{C}_{2} \mathrm{O}_{4}$ ). Without $\mathrm{Na}_{2} \mathrm{C}_{2} \mathrm{O}_{4}$, the $\mathrm{HNO}_{3}$ concentration is high, and the $C_{\mathrm{H}^{+}}$is about $50 \mathrm{mM}$. In this case there will be a large number of $\mathrm{Ag}$ seeds with stacking faults, resulting in the formation of Ag nanoflakes with a very high content of irregular $\mathrm{Ag}$ nanoparticles, see Fig. 4a. With the addition of $0.25 \mathrm{~mol}$ $\mathrm{Na}_{2} \mathrm{C}_{2} \mathrm{O}_{4}$, the $C_{\mathrm{H}^{+}}$becomes $33.3 \mathrm{mM}$, and the quantity of $\mathrm{Ag}$ nanoflakes with regular shapes increases considerably because of the reduced etching ability of $\mathrm{HNO}_{3}$, see Fig. $4 \mathrm{~b}$. When the amount of $\mathrm{Na}_{2} \mathrm{C}_{2} \mathrm{O}_{4}$ added is $0.5 \mathrm{~mol}$, the $C_{\mathrm{H}^{+}}$reduces to $16.7 \mathrm{mM}$, and the resultant $\mathrm{Ag}$ nanoflakes have regular shapes with very few $\mathrm{Ag}$ nanoparticulates, see Fig. 4c. When only $\mathrm{Na}_{2} \mathrm{C}_{2} \mathrm{O}_{4}$ is used without $\mathrm{HNO}_{3}$, the $C_{\mathrm{H}^{+}}$is zero, and the product consists of only $\mathrm{Ag}$ nanoparticles, see Fig. $4 \mathrm{~d}$. These results demonstrate that both too low and too high a $\mathrm{H}^{+}$concentration will result in the formation of Ag nanoparticles, and the highest yield of $\mathrm{Ag}$ nanoflakes can be achieved with a $\mathrm{H}^{+}$concentration of about $16.7 \mathrm{mM}$.

\section{Reaction temperature}

At room temperature, $\mathrm{Ag}_{2} \mathrm{C}_{2} \mathrm{O}_{4}$ is very stable, and the $\mathrm{Ag}^{+}$cannot be reduced because of the very weak reducing power of PVP.

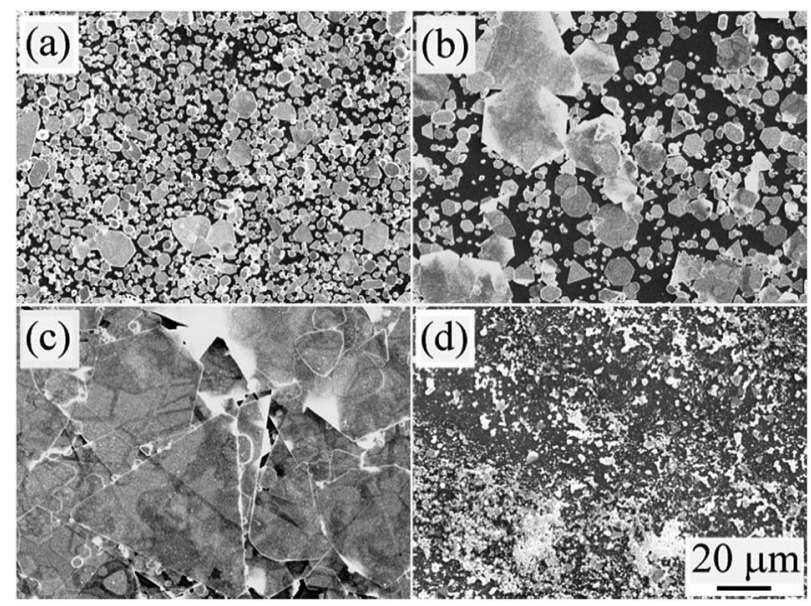

Fig. 4 FESEM images of the Ag nanoflakes at a $\mathrm{H}^{+}$concentration of (a) 50, (b) 33.3, (c) 16.7 and (d) $0 \mathrm{mM}$. 
Applying a certain temperature to promote the decomposition of $\mathrm{Ag}_{2} \mathrm{C}_{2} \mathrm{O}_{4}$ and the reduction of $\mathrm{Ag}^{+}$is indispensable. Fig. S3 $\uparrow$ shows the FESEM images of the products after reaction at different temperatures. One can see that the size of the product firstly increases, reaches a maximum value, and then decreases with an increasing temperature. The optimal temperature is $130{ }^{\circ} \mathrm{C}$, at which the product consists almost completely of $\mathrm{Ag}$

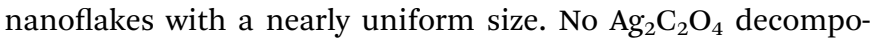
sition occurs at a temperature of $100{ }^{\circ} \mathrm{C}$, so the product is still $\mathrm{Ag}_{2} \mathrm{C}_{2} \mathrm{O}_{4}$, see Fig. S3a and g. $\dagger$ The thermal energy at a temperature of $110^{\circ} \mathrm{C}$ is still not high enough to decompose $\mathrm{Ag}_{2} \mathrm{C}_{2} \mathrm{O}_{4}$ at a reasonable rate, and the product consists of a small amount of $\mathrm{Ag}$ nanoflakes and a large quantity of irregular Ag nanoparticles, see Fig. S3b and g. $\uparrow$ The amount of irregular Ag nanoparticles decreases and the amount of $\mathrm{Ag}$ nanoflakes increases substantially with increasing temperature (Fig. S3c $\dagger$ ), and at a temperature of $130^{\circ} \mathrm{C}$, the product almost completely consists of nanoflakes, see Fig. S3d and g. $\dagger$ But if the temperature is over $130{ }^{\circ} \mathrm{C}$, spherical $\mathrm{Ag}$ nanoparticles will appear, and their amount increases with an increasing temperature, see Fig. S3e and $\mathrm{f} . \dagger$

The formation of the $\mathrm{Ag}$ nanoflakes depends on the concentration of $\mathrm{Ag}^{+}$in the system. At a low temperature the concentration of $\mathrm{Ag}^{+}$is relatively low, and thus the content of $\mathrm{Ag}$ nanoflakes in the product is low. On the other hand, low temperatures will favour the formation of the $\mathrm{Ag}$ crystal nuclei with multilayer stacking faults, and the Ag nanoflakes will have a high thickness. At a low $\mathrm{Ag}^{+}$concentration, some of the $\mathrm{Ag}$ crystal nuclei will be completely covered by PVP, leading to the formation of irregular $\mathrm{Ag}$ nanoparticles. At a temperature of $130{ }^{\circ} \mathrm{C}$, the thermal decomposition rate of $\mathrm{Ag}_{2} \mathrm{C}_{2} \mathrm{O}_{4}$ is optimal, and thus the products are all $\mathrm{Ag}$ nanoflakes. At temperatures higher than $130{ }^{\circ} \mathrm{C}$, the decomposition rate of $\mathrm{Ag}_{2} \mathrm{C}_{2} \mathrm{O}_{4}$ is very fast, and $\mathrm{Ag}^{+}$will quickly transform to $\mathrm{Ag}$, resulting in the formation of spherical $\mathrm{Ag}$ nanoparticles.

\section{Reaction time}

Fig. 5 shows the FESEM images of the products after reaction for different times. The initial white precipitation shown in Fig. $5 \mathrm{a}$ is $\mathrm{Ag}_{2} \mathrm{C}_{2} \mathrm{O}_{4}$, as proven by the corresponding XRD analysis shown in Fig. $5 \mathrm{i}$, in which the diffraction peak can be indexed to monoclinic $\mathrm{Ag}_{2} \mathrm{C}_{2} \mathrm{O}_{4}$ (JCPDS card no. 22-1335) without the presence of other phases, indicating that the metallic silver exists in the form of Ag-oxalate. After reaction for 0.5 and $1 \mathrm{~h}$, the $\mathrm{Ag}_{2} \mathrm{C}_{2} \mathrm{O}_{4}$ began to decompose slowly, the amount of $\mathrm{Ag}_{2} \mathrm{C}_{2} \mathrm{O}_{4}$ decreased, and there were some flake-like products, as shown in Fig. $5 \mathrm{~b}$ and $\mathrm{c}$, with the corresponding XRD patterns showing a reduced peak intensity from the $\mathrm{Ag}_{2} \mathrm{C}_{2} \mathrm{O}_{4}$ signal and an increased peak intensity from the FCC $\mathrm{Ag}$ signal. When the reaction time was increased to $2 \mathrm{~h}$, the product mainly consisted of $\mathrm{Ag}$ nanoflakes without the presence of $\mathrm{Ag}_{2} \mathrm{C}_{2} \mathrm{O}_{4}$, as shown in Fig. $5 \mathrm{~d}$ and $\mathrm{i}$. With a further increase in reaction time to $3 \mathrm{~h}$ and above, the products are $\mathrm{Ag}$ nanoflakes, and their size increases with increasing reaction time, as shown in Fig. 5e-g. After further increases in reaction time to $8 \mathrm{~h}$, there are no
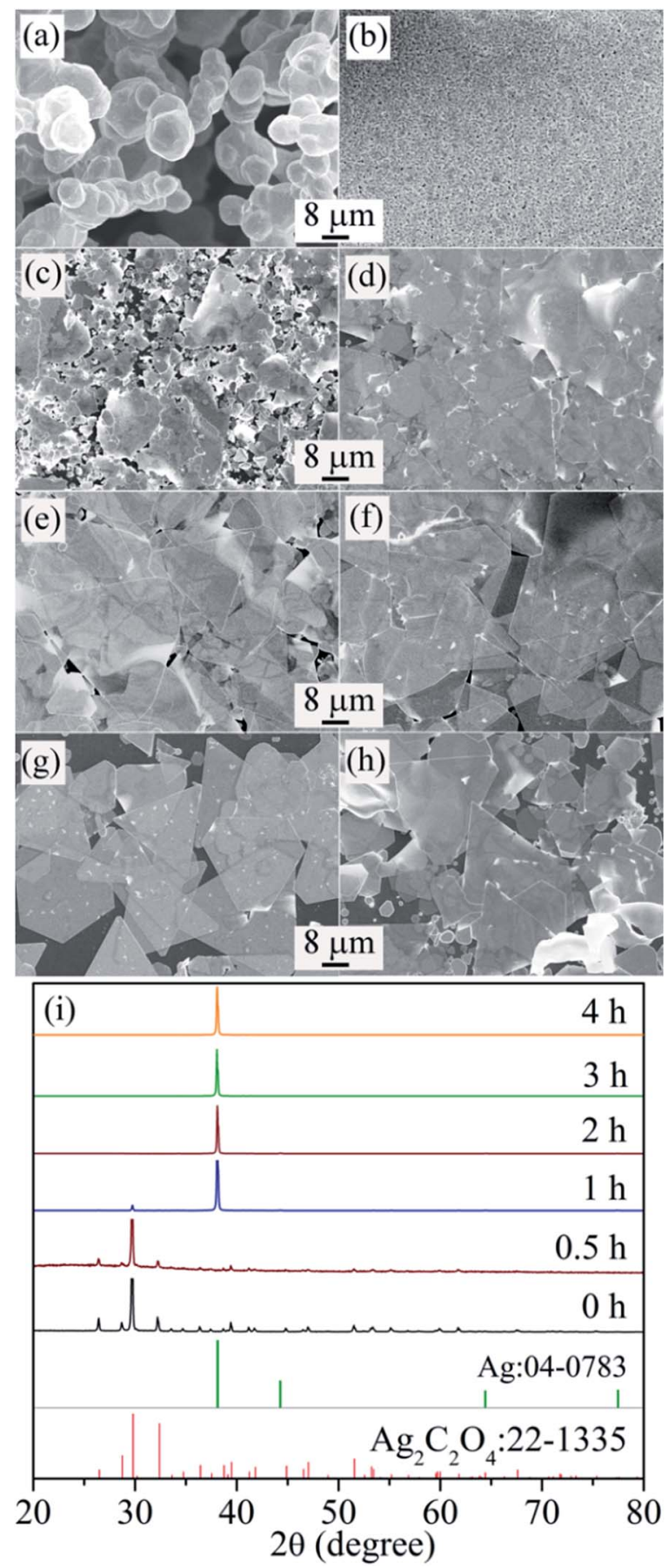

Fig. 5 The FESEM images of the products at a reaction time of (a) 0 , (b) 0.5, (c) 1, (d) 2, (e) 3, (f) 4, (g) 5 and (h) 8 h. (i) The corresponding XRD spectra of the products after reaction for different times.

changes in either the size or thickness of the Ag nanoflakes, indicating that the entire $\mathrm{Ag}$ source has been consumed.

Based on the above analysis, one can see that a large output of $\mathrm{Ag}$ nanoflakes can be realized under carefully controlled hydrothermal conditions. To verify this, the mass of PVP-K60, $\mathrm{AgNO}_{3}, \mathrm{H}_{2} \mathrm{C}_{2} \mathrm{O}_{4}$ and $\mathrm{Na}_{2} \mathrm{C}_{2} \mathrm{O}_{4}$ reactants were respectively increased to $12 \mathrm{~g}, 2.55 \mathrm{~g}, 409.5 \mathrm{mg}$ and $569.5 \mathrm{mg}$, and the volume of deionized water was fixed at $390 \mathrm{~mL}$. After 
hydrothermal reaction at $130{ }^{\circ} \mathrm{C}$ for $10 \mathrm{~h}$, the total output of the $\mathrm{Ag}$ nanoflakes was about $1.5 \mathrm{~g}$, as shown in Fig. $\mathrm{S} 4, \dagger$ which is about 10 times larger than that shown in Fig. 3b. From Fig. S4† one also can see that the Ag nanoflakes have a high aspect ratio, despite their different shapes. The maximum size of the Ag nanoflakes is about $60 \mu \mathrm{m}$ with a thickness of about $75.5 \mathrm{~nm}$ and an aspect ratio of about 800 . This result demonstrates that even a large-scale production of $\mathrm{Ag}$ nanoflakes can be implemented by further increasing the amount of the reactants.

Based on the above discussions, the growth mechanism of the Ag nanoflakes can be summarised as follows. Firstly, during the initial stage, $\mathrm{Ag}$ twin seeds with stacking faults were formed in an aqueous colloidal system of PVP under hydrothermal conditions, which is essential for the growth of the Ag nanoflakes. Following this, these twin seeds gradually grew into small Ag nanoflakes under the induction of PVP, and the Ag atoms continuously moved onto the nucleation sites with reentrant groove structures which facilitated subsequent lateral crystal growth. Meanwhile, the Ag flakes also grew along the longitudinal direction, but their growth rate in this direction was much slower than in the lateral direction. Finally, Ag nanoflakes with a lateral size of several tens of micrometers and aspect ratios of several hundred were formed. It is worth noting that Ag nanoflakes with a regular hexagonal shape are the main products in a uniform, closed and stable experimental environment, while Ag nanoflakes of different shapes will be formed under unstable hydrothermal conditions.

Due to its optimal electrical and thermal conductivity as well as its excellent chemical stability, metal $\mathrm{Ag}$ has been widely used as a conductive phase in conductive pastes. Currently the conductive fillers in commercial $\mathrm{Ag}$ conductive pastes are mostly granular Ag obtained mainly by a ball-milling method. The conductivity of a granular Ag conductive phase is generally low because of the point contact between Ag particles, and the electronic channels between $\mathrm{Ag}$ particles are narrow, thereby affecting electron transportation and the electrical conductivity. A poor crystallinity and the adherence of certain dispersants to the surface of $\mathrm{Ag}$ particles also have a great impact on the conductivity of the $\mathrm{Ag}$ conductive pastes. On the other hand, the two dimensional $\mathrm{Ag}$ single crystalline nanoflakes with a large surface area will have both linear and face-to-face contact, leading to a high conductivity if used as conductive fillers in commercial Ag pastes. Ag nanowires have been frequently used to build flexible film heaters, ${ }^{\mathbf{4 1 - 4 4}}$ and it has been found that there is fusing of the wire-to-wire junctions; as a result, pretreatment of the $\mathrm{Ag}$ nanowires is needed before use. $\mathrm{Ag}$ nanoflakes have a high conductivity and could also be excellent fillers in the construction of flexible film heaters. In the following paragraphs, we will study the thermal properties and conductivity of the $\mathrm{Ag}$ nanoflakes.

Fig. 6 shows the surface temperature of a $\mathrm{Ag}$ nanoflake film heater $\left(R_{\mathrm{S}}=1.1 \Omega \mathrm{sq}^{-1}\right)$ operated at different input voltages. The heater was fabricated by drop-casting a $\mathrm{Ag}$ nanoflake ethanol solution with a size of $25-40 \mu \mathrm{m}$ onto a PET substrate without the addition of an adhesion agent. It was found that the nanoflakes have excellent bonding between themselves after annealing at $180{ }^{\circ} \mathrm{C}$ for $0.5 \mathrm{~h}$ in air. The input voltages were

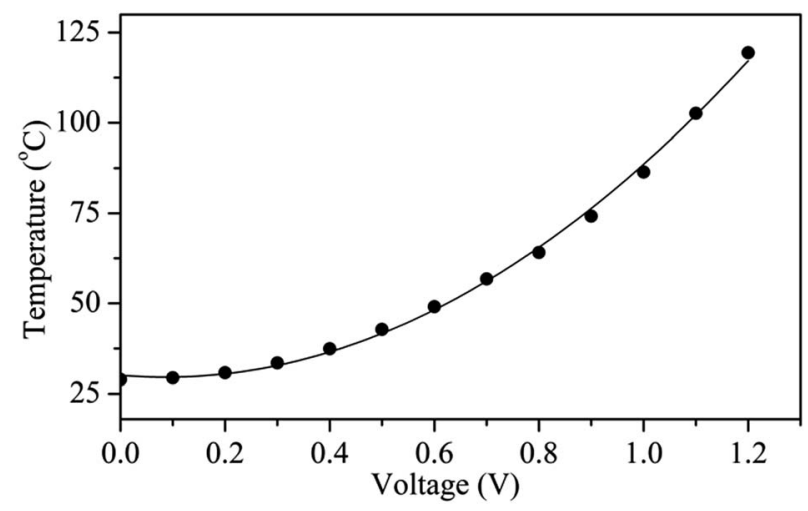

Fig. 6 The surface temperature of the Ag nanoflake heater under different input voltages.

supplied to the heater through two electrodes on the edges of the $\mathrm{Ag}$ nanoflake film. From Fig. 6 one can clearly see that the heating temperature non-linearly increases with an increasing input voltage (with the fitting function of $T=30.1-12 \mathrm{~V}+$ $70.6 V^{2}$ ), which is in accordance with the previous reports. ${ }^{45,46}$ Fig. 7 shows the temperature profiles of the $\mathrm{Ag}$ nanoflake heater operated at different voltages. One can see that the temperature increases rapidly and reaches its saturation values within $30 \mathrm{~s}$; the higher the input voltage the higher the temperature. The temperature at $1.1 \mathrm{~V}$ can reach as high as about $100{ }^{\circ} \mathrm{C}$, which is much higher than that of the $\mathrm{Ag}$ nanowire heaters reported in the literature. ${ }^{\mathbf{4 1 , 4 2 , 4 4}}$ Infrared photography of the film heater at a constant input voltage (Fig. S5†) clearly revealed that the temperature distribution on the film area was uniform, and that the temperature is about 43.1 and $62.8^{\circ} \mathrm{C}$ at a voltage of 0.5 and $0.8 \mathrm{~V}$, respectively. Cyclic temperature testing confirmed that the Ag nanoflake heater has a high thermal stability, and no fusing phenomenon was observed on the flake-to-flake junctions (Fig. S6 $\dagger$ ). The heat power is affected by the bias and the resistance of the heater, and due to the direct formation of a face-to-face contact conductive network between the $\mathrm{Ag}$ nanoflakes, the square resistance of the $\mathrm{Ag}$ nanoflake film

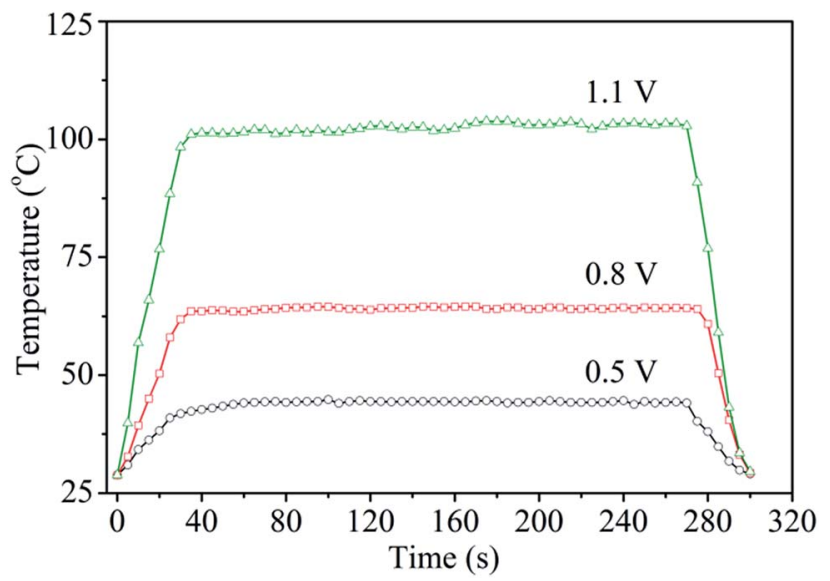

Fig. 7 The temperature profiles of the Ag nanoflake film heater operated at different voltages. 


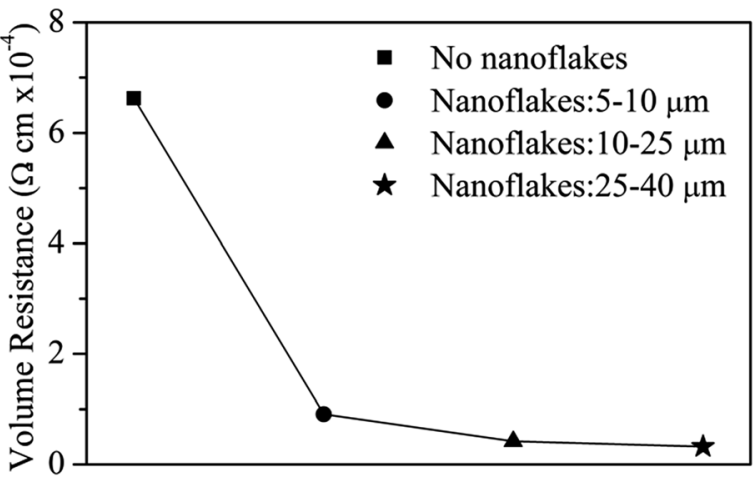

Fig. 8 The volume resistivity of Ag conductive pastes without and with addition of Ag nanoflakes of different sizes.

heater is very low, only about $1.1 \Omega \mathrm{sq}^{-1}$, and thus the temperature of the $\mathrm{Ag}$ nanoflake film heater is higher than that of the $\mathrm{Ag}$ nanowire/PVP film heater at the same input voltage. ${ }^{41}$

Ag nanoflakes with a size of 5-10, 10-25 and $25-40 \mu \mathrm{m}$ were mixed with a commercial $\mathrm{Ag}$ particle paste with a $1: 7$ mass ratio of nanoflake/particle, forming a hybrid Ag paste. Fractions of $\mathrm{Ag}$ nanoflakes of different sizes were isolated using ethanolinduced precipitation with the aid of ultrasound. Fig. 8 shows the volume resistance of the hybrid Ag pastes together with the original Ag paste. One can see that the resistance decreases as the size of the Ag nanoflakes increases, and the larger the size of the Ag nanoflakes, the lower the resistance, which is in good agreement with a previous report. ${ }^{33}$ The conductivity of the hybrid Ag paste increased by more than an order of magnitude with the addition of $\mathrm{Ag}$ nanoflakes. Nevertheless, if there is a porous structure after combining the Ag nanoparticles with the $\mathrm{Ag}$ flakes, the conductivity of the hybrid $\mathrm{Ag}$ paste will decrease with an increase in silver flake content. ${ }^{47}$ We have not observed this phenomenon, which provides further evidence that the Ag silver flakes prepared in this work are of high quality. A larger contact area and more electronic channels between the $\mathrm{Ag}$ nanoflakes are responsible for the reduced resistance. The bridging role of the $\mathrm{Ag}$ nanoflakes in the original $\mathrm{Ag}$ particles also contributes to the enhanced conductivity of the hybrid pastes. These results indicate that highly conductive Ag pastes can be achieved by simply adding a small amount of $\mathrm{Ag}$ nanoflakes into a commercial $\mathrm{Ag}$ paste. The optimal size and amount to be added need further optimization.

\section{Conclusions}

In conclusion, Ag nanoflakes with a size of 5-60 $\mu \mathrm{m}$, thickness of $20-80 \mathrm{~nm}$ and aspect ratio of up to 800 have been synthesized via a simple hydrothermal method. The kinetic growth process of the Ag nanoflakes can be well controlled by introducing an oxalate compound in the form of $\mathrm{Ag}_{2} \mathrm{C}_{2} \mathrm{O}_{4}$, causing the slow release of $\mathrm{Ag}^{+}$at a temperature of $130{ }^{\circ} \mathrm{C}$. It was found that the polymerization degree and concentration of $\mathrm{PVP}, \mathrm{H}^{+}$concentration, reaction time and reaction temperature all affect the formation of the $\mathrm{Ag}$ nanoflakes, and that controlling the $\mathrm{H}^{+}$ concentration is essential in the formation of Ag crystal nuclei with a stacking fault. Our method takes advantage of PVP as both a capping agent to induce anisotropic growth of the $\mathrm{Ag}$ nanoflakes and as a reducing agent to reduce the $\mathrm{Ag}^{+}$to $\mathrm{Ag}$, and can be easily scaled up for mass production of the Ag nanoflakes. A large contact area with more electronic channels between the Ag nanoflakes means that the Ag nanoflakes are excellent conductive fillers for the fabrication of flexible film heaters and high conductivity pastes.

\section{Experimental section}

\section{Materials}

Silver nitrate $\left(\mathrm{AgNO}_{3}, 99 \%\right)$, sodium oxalate $\left(\mathrm{Na}_{2} \mathrm{C}_{2} \mathrm{O}_{4}, 99.8 \%\right)$, oxalic acid $\left(\mathrm{H}_{2} \mathrm{C}_{2} \mathrm{O}_{4}, 99.5 \%\right)$, polyvinylpyrrolidone (PVP, K30, $\mathrm{K} 60, \mathrm{~K} 90)$ and ethanol (99.7\%) were purchased from Sinopharm Chemical Reagent Co. Ltd. All chemicals were of analytical grade and used as received without further purification. Silver conductive paste $(70 \%)$ was purchased from PuQiang (Suzhou) Conductive Paints Co., Ltd. Triply distilled water (1.82 M $\Omega$ ) was used in all experiments.

\section{Synthesis}

In a typical synthesis process of the $\mathrm{Ag}$ nanoflakes, an aqueous solution of PVP-K60 $(0.54 \mathrm{M}, 20 \mathrm{~mL}), \mathrm{H}_{2} \mathrm{C}_{2} \mathrm{O}_{4}(0.25 \mathrm{M}, 1 \mathrm{~mL})$, $\mathrm{Na}_{2} \mathrm{C}_{2} \mathrm{O}_{4}(0.25 \mathrm{M}, 1 \mathrm{~mL})$ and $\mathrm{AgNO}_{3}(0.5 \mathrm{M}, 3 \mathrm{~mL})$ was firstly mixed in a glass beaker and stirred for half an hour; the obtained suspended-precipitate solution was then poured into a $50 \mathrm{~mL}$ Teflon-lined autoclave container. The Teflon container was sealed and kept at $130{ }^{\circ} \mathrm{C}$ in an oven for $3 \mathrm{~h}$. Finally, the products were obtained by centrifuging the final reaction solution, and washed with ethanol and/or water 4 times at a centrifugal speed of $1500 \mathrm{rpm}$ for $5 \mathrm{~min}$, and dried at $60{ }^{\circ} \mathrm{C}$. The nanoflakes were dispersed in ethanol for storage.

\section{Characterization}

The morphology and size of the products were characterized using field-emission scanning electron microscopy (FESEM, Sirion 200) and atomic force microscopy (AFM, Innova). The structure and the crystalline phases were analyzed using transmission electron microscopy (TEM, JEOL Model 2010) and X-ray diffraction with a $\mathrm{Cu} \mathrm{K} \alpha$ line (XRD, Philips X'Pert). The resistance of conductive paste was measured with a 4 points method using a Keithley digital source meter (2612A). The surface temperature of the heater was monitored using an infrared radiation thermometer (TES-1310). Infrared photographs of the heater were taken at a constant input voltage with an IR-Fusion Thermal Imager (Ti200).

\section{Conflicts of interest}

There are no conflicts to declare. 


\section{Acknowledgements}

This work was financially supported by the National Basic Research Program of China (Grant no. 2013CB934304) and in part by Yantai Shied Advanced Materials Co., LTD.

\section{Notes and references}

1 E. Borsella, E. Cattaruzza, G. De Marchi, F. Gonella, G. Mattei, P. Mazzoldi, A. Quaranta, G. Battaglin and R. Polloni, J. Non-Cryst. Solids, 1999, 245, 122-128.

2 S. H. Jeong, H. Choi, J. Y. Kim and T. W. Lee, Part. Part. Syst. Charact., 2015, 32, 164-175.

3 K. Kim, K. Hong, B. Koo, I. Lee and J. L. Lee, Appl. Phys. Lett., 2013, 102, 3511-3514.

4 B. Park, I. G. Bae and Y. H. Huh, Sci. Rep., 2016, 6, 19485.

5 J. A. Suarez, J. J. Plata, A. M. Marquez and J. F. Sanz, Theor. Chem. Acc., 2016, 135, 1-8.

6 F. Hajakbari and F. Shafieinejad, Jpn. J. Appl. Phys., 2016, 55, 035503.

7 C. H. Zhu, G. W. Meng, P. Zheng, Q. Huang, Z. B. Li, X. Y. Hu, X. J. Wang, Z. L. Huang, F. D. Li and N. Q. Wu, Adv. Mater., 2016, 28, 4871-4876.

8 Y. Xie, M. Zhao, Q. Hu, Y. Cheng, Y. Guo, H. Qian and W. Yao, J. Raman Spectrosc., 2017, 48, 204-210.

9 H. B. Tang, P. Zheng, G. W. Meng, Z. B. Li, C. H. Zhu, F. M. Han, Y. Ke, Z. M. Wang, F. Zhou and N. Q. Wu, Nanotechnology, 2016, 27, 325303-325317.

10 Z. B. Li, G. W. Meng, Q. Huang, C. H. Zhu, Z. Zhang and X. D. Li, Chem.-Eur. J., 2012, 18, 14948-14953.

11 E. L. Holthoff, D. N. Stratis-Cullum and M. E. Hankus, Sensors, 2011, 11, 2700-2714.

12 H. J. Jiang, K. S. Moon, Y. Li and C. P. Wong, Chem. Mater., 2006, 18, 2969-2973.

13 H. W. Cui, Q. Fan and D. S. Li, Int. J. Adhes. Adhes., 2014, 48, 177-182.

14 R. W. Zhang, W. Lin, K. Lawrence and C. P. Wong, Int. J. Adhes. Adhes., 2010, 30, 403-407.

15 Y. H. Ji, Y. Liu, G. W. Huang, X. J. Shen, H. M. Xiao and S. Y. Fu, ACS Appl. Mater. Interfaces, 2015, 7, 8041-8052.

16 H. M. Ren, Y. Guo, S. Y. Huang, K. Zhang, M. M. F. Yuen, X. Z. Fu, S. H. Yu, R. Sun and C. P. Wong, ACS Appl. Mater. Interfaces, 2015, 7, 13685-13692.

17 S. Kiruthika, R. Gupta and G. U. Kulkarni, $R S C A d v ., 2014,4$, 49745-49751.

18 P. H. Wang, S. P. Chen, C. H. Su and Y. C. Liao, RSC Adv., 2015, 5, 98412-98418.

19 K. D. Kim, K. Y. Choi and H. T. Kim, Scr. Mater., 2005, 53, 571-575.

20 Y. G. Sun, Y. D. Yin, B. T. Mayers, T. Herricks and Y. N. Xia, Chem. Mater., 2002, 14, 4736-4745.

21 M. Luo, H. W. Huang, S. I. Choi, C. Zhang, R. R. da Silva, H. C. Peng, Z. Y. Li, J. Y. Liu, Z. K. He and Y. N. Xia, ACS Nano, 2015, 9, 10523-10532.
22 Y. Wang, Y. Q. Zheng, C. Z. Huang and Y. N. Xia, J. Am. Chem. Soc., 2013, 135, 1941-1951.

23 X. X. Liu, L. L. Li, Y. D. Yang, Y. D. Yin and C. B. Gao, Nanoscale, 2014, 6, 4513-4516.

24 Q. Y. Li, S. C. Liu, S. R. Li, W. H. Guo and C. F. Wu, J. Mater. Sci.: Mater. Electron., 2016, 27, 452-457.

25 H. Wang, H. Wu, L. Zhong, J. Zhao and G. Li, J. Electrochem. Soc., 2017, 164, D225-D229.

26 L. P. Jiang, S. Xu, J. M. Zhu, J. R. Zhang, J. J. Zhu and H. Y. Chen, Inorg. Chem., 2004, 43, 5877-5883.

27 D. P. Chen, X. G. Zhu, G. Zhu, X. L. Qiao and J. G. Chen, J. Mater. Sci.: Mater. Electron., 2012, 23, 625-630.

28 A. X. Zhai, X. H. Cai and B. Du, Trans. Nonferrous Met. Soc. China, 2014, 24, 1452-1457.

29 R. C. Jin, Y. W. Cao, C. A. Mirkin, K. L. Kelly, G. C. Schatz and J. G. Zheng, Science, 2001, 294, 1901-1903.

30 H. Chen, F. Simon and A. Eychmuller, J. Phys. Chem. C, 2010, 114, 4495-4501.

31 H. Z. Liang, D. J. Kim, H. S. Chung, J. Zhang, K. N. Yu, S. H. Li and R. X. Li, Acta Phys.-Chim. Sin., 2003, 19, 150-153.

32 Z. Deng, M. Mansuipur and A. J. Musca, J. Phys. Chem. C, 2009, 113, 867-873.

33 Y. M. Park, B. G. Lee, J.-I. Weon and M. H. Kim, RSC Adv., 2016, 6, 95768-95773.

34 D. Aherne, D. M. Ledwith, M. Gara and J. M. Kelly, Adv. Funct. Mater., 2008, 18, 2005-2016.

35 V. Germain, J. Li, D. Ingert, Z. L. Wang and M. P. Pileni, J. Phys. Chem. B, 2003, 107, 8717-8720.

36 J. L. Song, Y. Chu, Y. Liu, L. L. Li and W. D. Sun, Chem. Commun., 2008, 1223-1225.

37 K. M. Koczkur, S. Mourdikoudis, L. Polavarapu and S. E. Skrabalak, Dalton Trans., 2015, 44, 17883-17905.

38 M. H. Kim, D. K. Yoon and S. H. Im, RSC Adv., 2015, 5, 14266-14272.

39 Y. J. Xiong, I. Washio, J. Y. Chen, H. G. Cai, Z. Y. Li and Y. N. Xia, Langmuir, 2006, 22, 8563-8570.

40 C. S. Wang, C. X. Kan, J. J. Zhu, X. L. Zeng, X. F. Wang, H. C. Li and D. N. Shi, J. Nanomater., 2010, 1, 139-143.

41 W. Lan, Y. X. Chen, Z. W. Yang, W. H. Han, J. Y. Zhou, Y. Zhang, J. Y. Wang, G. M. Tang, Y. P. Wei, W. Dou, Q. Su and E. Q. Xie, ACS Appl. Mater. Interfaces, 2017, 9, 6644-6651.

42 K. H. Pyo and J. W. Kim, Compos. Sci. Technol., 2016, 133, 714.

43 M. Li, S. L. Ji, J. Pan, H. Wu, L. Zhong, Q. Wang, F. D. Li and G. H. Li, J. Mater. Chem. A, 2014, 2, 20470-20473.

44 R. Gupta, K. D. M. Rao, S. Kiruthika and G. U. Kulkarni, ACS Appl. Mater. Interfaces, 2016, 8, 12559-12575.

45 Q. J. Huang, W. F. Shen, X. Z. Fang, G. F. Chen, J. C. Guo, W. Xu, R. Q. Tan and W. J. Song, RSC Adv., 2015, 5, 4583645842 .

46 J. P. Li, J. J. Liang, X. Jian, W. Hu, J. Li and Q. B. Pei, Macromol. Mater. Eng., 2014, 299, 1403-1409.

47 B. G. Park, K. H. Jung and S. B. Jung, J. Alloys Compd., 2017, 699, 1186-1191. 